\title{
Current Urology
}

We would like to express our great appreciation to all the following reviewers who served Current Urology for the first volume since the inaugural issue of September 2007. We gratefully acknowledge their generous, knowledgeable, and constructive contribution.

Yilmaz Akgun, Diyarbakir, Turkey

Mayank M. Agarwal, Chandigarh, India

Piero L Alo, Rome, Italy

João L. Amaro, Botucatu, Brazil

Jean Amiel, Nice, France

Patrizia Angelico, Milano, Italy

Ninaad S. Awsare, Stevenage, United Kingdom

Chandrasekhar Bal, New Delhi, India

Guido Barbagli, Arezzo, Italy

Neil J. Barber, London, United Kingdom

Joseph G. Barone, New Brunswick, USA

John M. Barry, Portland, USA

Anthony J. Bella, Ottawa, Canada

John M. Bennett, Lexington, USA

Chandra S. Biyani, Wakefield, United Kingdom

Marco H. Blanker, ZR Hattem, The Netherlands

Alberto Breda, Los Angeles, USA

Nicola Capozza, Rome, Italy

Pasquale Casale, Philadelphia, USA

Liang Cheng, Indianapolis, USA

Rocco Damiano, Catanzaro, Italy

Christopher J. Danpure, London, United Kingdom

Andre A de Figueiredo, Sao Paulo, Brazil

Aslan Demir, Istanbul, Turkey

John D. Denstedt, London, United Kingdom

Antonio Dessanti, Sassari, Italy

Harpreet K. Dhiman, New Delhi, India

Lars Egevad, Lyon, France

Scott Eggener, New York, USA

Paulo H. Egydio, Sao Paulo, Brazil

Michael J. Englesbe, Ann Arbor, USA

Aristomenis K. Exadaktylos, Berne, Switzerland

Aaron Fenster, London, United Kingdom

Pedro C. Ferreira, Barcelos, Portugal

Vincenzo Ficarra, Verona, Italy

Alessandro Frati, Rome, Italy

Qiang Fu, Shanghai, China

Markus Giessing, Berlin, Germany
Stavros Gravas, Larissa, Greece

Jan Groen, Rotterdam, The Netherlands

Andreas J. Gross, Hamburg, Germany

Harry W. Herr, New York, USA

Andreas Hinkel, Herne, Germany

Piet B. Hoebeke, Ghent, Belgium

Matthew Hotston, Bristol, United Kingdom

Don A. Hudson, Cape Town, South Africa

Günter Janetschek, Linz, Austria

Steven Joniau, Leuven, Belgium

Klaus Jung, Berlin, Germany

John Kavanagh, Manchester, United Kingdom

Thomas M. Kessler, Bern, Switzerland

Saeed Khan, Gainesville, USA

Mustafa Kibar, Adana, Turkey

Adam P. Klausner, Charlottesville, USA

Rainer M. Kuntz, Berlin, Germany

Jean-Jacques Labat, Nantes, France

Craig B. Langman, Chicago, USA

Robert M. Levin, Albany, USA

Borje Ljungberg, Umeå, Sweden

Simon S. Lo, Columbus, USA

Marios Loukas, Boston, USA

Tom F. Lue, San Francisco, USA

Danil V. Makarov, Baltimore, USA

Shane K. Maloney, Crawley, Australia

Matthias May, Cottbus, Germany

Gregor Mikuz, Innsbruck, Austria

Yu Ming, Stockholm, Sweden

Rodolfo Montironi, Ancona, Italy

Alvaro Morales, Kingston, Canada

Edgar M. Moran, Irvine, USA

Hasan Mukhtar, Madison, USA

Michael A. O'Donnell, Iowa City, USA

Jacob ST. Pang, Tao Yuan, Taiwan

Juergen Pannek, Herne, Germany

Athanasios G. Papatsoris, London, United Kingdom

Gyan Pareek, Providence, USA
(ㄷ) 2007 S. Karger AG, Basel

Fax +4161306 1234

E-Mail karger@karger.ch

www.karger.com

Accessible online at:

www.karger.com/cur 
Sangtae Park, Seattle, USA

Beth R. Pflug, Pittsburgh, USA

Achille Pich, Turin, Italy

Robert Pickard, Newcastle-upon-Tyne, United Kingdom

David Ramos, Valencia, Spain

Simmi K. Ratan, New Delhi, India

Allen L. Rodgers, Cape Town, South Africa

Claus G. Roehrborn, Dallas, USA

Abdul M. Rona, Karachi, Pakistan

Mark A. Rosen, Philadelphia, USA

Morgan Roupret, Paris, France

Paul Russo, New York, USA

Farid Saad, Berlin, Germany

Kemal Sarica, Istanbul, Turkey

Mitsuyoshi Sasaki, Tokyo, Japan

Markus H. Schmidt, Dublin, USA

Pejman Shadpour, Tehran, Iran

Ahmed Shafik, Cairo, Egypt

Hemendra N. Shah, Maharashtra, India

Andrew I. Shpall, Los Angeles, USA

Akhouri A. Sinha, Minneapolis, USA

Mario Sofer, Tel Aviv, Israel

Prasanna Sooriakumaran, Surrey, United Kingdom

Viktor Soukup, Prague, Czeck Republic

John P. Stein, Los Angeles, USA

Jens-Uwe Stolzenburg, Leipzig, Germany

Tomi K. Streng, Lund, Sweden
Peter Sun, Indianapolis, USA

Al Taira, Seattle, USA

Yeh Hong Tan, Singapore, Singapore

Afshin Tavakoli, Manchester, United Kingdom

Joel M. Teichman, Vancouver, Canada

Khai-Lee Toh, Singapore, Singapore

Kevin M. Tomera, Anchorage, USA

Christoph Troppmann, Sacramento, USA

Yasuhiro Tsukimi, Kyoto, Japan

Jyoti Upadhyay, Syracuse, USA

Frank van der Aa, Leuven, Belgium

Bas W. van Rhijn, Rotterdam, The Netherlands

Robert W. Veltri, Baltimore, USA

Peter Veranic, Ljubljana, Slovenia

Nado Vodopija, Slovenj Gradec, Slovenia

Run Wang, Houston, USA

Emily Ward, Dublin, Ireland

Marcia A. Wheeler, New Haven, USA

Hessel Wijkstra, Amsterdam, The Netherlands

Chad T. Wilson, White River Junction, USA

J. Alfred Witjes, Nijmegen, The Netherlands

J. Stuart Wolf Jr., Ann Arbor, USA

Ugur Yilmaz, Seattle, USA

Sidney K. Yip, Hong Kong, China

Mitsuharu Yoshiyama, Yamanashi, Japan

Peng Zhai, Houston, USA 